\title{
Distribution and social determinants of overweight and obesity: a cross-sectional study of non-pregnant adult women from the Malawi Demographic and Health Survey (2015-2016)
}

\author{
Leonard Mndala ${ }^{1,2}$, Abhay Kudale ${ }^{2}$ \\ 'Complete Health Foundation, Lilongwe, Malawi; Interdisciplinary School of Health Sciences, Savitribai Phule Pune University, Pune, India
}

\begin{abstract}
OBJECTIVES: Hitherto regarded as a public health issue of well-heeled nations, overweight and obesity have emerged as a problem of concern in developing nations. Although social and demographic factors are equally important as proximal lifestyle factors affecting health, their role is neither well researched nor well understood. We conducted a novel study to determine the distribution, prevalence, and social and demographic determinants of overweight/obesity in Malawi.
\end{abstract}

METHODS: A population-based, quantitative cross-sectional study using data from the Malawi Demographic and Health Survey (2015-2016) was conducted among non-pregnant women aged 18-49 years. A total of 6,443 women were included in the analysis. Overweight/obesity, defined as a body mass index (BMI) $\geq 25.0 \mathrm{~kg} / \mathrm{m}^{2}$, was the main outcome variable. The analysis was done in SPSS version 20.0; after calculating descriptive statistics, bivariate and multivariate logistic regression was conducted to evaluate associations and determine odds.

RESULTS: In total, $16.8 \%$ and $6.3 \%$ of women were overweight and obese, respectively $(\mathrm{p}<0.001)$. Overweight and obesity were more prevalent in urban than in rural areas. The BMI distribution among women varied across different background characteristics. Women from the Ngoni ethnicity were more likely to be overweight/obese than others (adjusted odds ratio [aOR], 1.54; $95 \%$ confidence interval [CI], 1.14 to 2.08). Socioeconomic status (SES) and the age of the respondent were highly significant determinants that were strongly associated with being overweight/obese. The richest women were 3 times more likely to be overweight/obese than the poorest (aOR, 3.30; 95\% CI, 2.46 to 4.43 ).

CONCLUSIONS: Overweight and obesity were highly prevalent and significantly associated with increasing SES, age, and being from the Ngoni ethnicity. Holistic interventions should also focus on improving social determinants in order to entirely curb the epidemic.

KEY WORDS: Overweight, Obesity, Malawian non-pregnant adult women, Body mass index, Social and demographic determinants, Cross-sectional studies

Correspondence: Leonard Mndala

Complete Health Foundation, P.O. Box 3206, Lilongwe, Malawi

E-mail: leonardmndala@gmail.com

Received: Jun 22, 2019 / Accepted: Sep 27, 2019 / Published: Sep 27, 2019

This article is available from: http://e-epih.org/

(c) This is an open-access article distributed under the terms of the Creative Commons Attribution License (http://creativecommons.org/licenses/by/4.0/), which permits unrestricted use, distribution, and reproduction in any medium, provided the original work is properly cited.

(C) 2019, Korean Society of Epidemiology

\section{INTRODUCTION}

In 2016, 2 billion adults aged 18 years and above were overweight, of whom 650 million were obese [1]. Hitherto regarded as a public health issue of well-heeled nations, overweight and obesity have also emerged as a problem of concern in developing low-income and middle-income countries (LMICs) [2]. The last decade has witnessed a noteworthy change in patterns of disease. This change was initially identified by Omran, and it has come to 
be known as the epidemiologic transition [3]. Tackling overweight and obesity is in accordance with attaining Sustainable Development Goal number 3 (SDG 3) for "Good Health and Wellbeing."

The World Health Organization (WHO) has prioritized "halting" the rise of obesity by the year 2025 in one of its 9 global voluntary targets to attain success in the fight against noncommunicable diseases (NCDs) [4]. The mounting epidemic in people of all age groups translates into an increasing risk of developing various NCDs, which are responsible for $70 \%$ of global mortality [5]. Overweight and obesity are established risk factors for cardiovascular disease (mainly stroke and heart disease), diabetes, musculoskeletal disorders, and many other forms of cancer. Apart from the resultant increased risk of mortality, overweight and obesity push people into poverty globally (especially in LMICs) through associated direct and indirect health care costs [6].

For the past decades, sub-Saharan Africa has been battling against undernutrition and infectious diseases, but in recent years, overweight and obesity and NCDs have put pressure on healthcare systems in this region [7]. The increase in rates of overweight and obesity has occurred more quickly in developing countries, thus translating into a "double-burden of malnutrition," as overweight and obesity exist side by side with undernutrition. Because overweight and obesity are established risk factors for NCDs, there has also been a "double burden" of persisting infectious diseases and emerging NCDs [8].

Numerous recommendations have been made for global surveillance of modifiable proximal determinants of overweight and obesity, but less attention has been paid to social factors, which in public health are equally important in the holistic understanding of various determinants of health [9]. As health is a social construct, the WHO's Commission on Social Determinants of Health has set up a comprehensive framework for understanding the different social factors that are associated with health. The framework brings together different aspects of social productivity in health and looks at social context, social stratification, differential exposure and vulnerabilities, and the consequences of ill health [10]. In this framework, behavior, biological, psychosocial factors, and other factors are regarded as social-cohesion factors. In research, although certain behaviors are studied as "lifestyle" factors, the social aspect of this framework stipulates that such factors and even biological factors (such as parity) are embedded within it.

The paucity of disaggregated information on the distribution of overweight and obesity based on various social factors and how such factors influence these conditions in women could mask the seriousness of overweight and obesity in adult non-pregnant women of Malawi. Therefore, research is needed to develop effective, context-specific, and population-based interventions for the prevention of obesity and NCDs. Developing targeted interventions for women based on relevant evidence would also translate into cost-effectiveness. The purpose of this study was to determine the prevalence and social and demographic determinants of overweight and obesity in adult non-pregnant women aged 18-49 years in Malawi using data from the Malawi Demographic and Health Survey (MDHS) 2015-2016.

\section{MATERIALS AND METHODS}

\section{Study population}

This cross-sectional study was conducted using data from the 2015-2016 MDHS. The survey is part of the global Demographic and Health Survey (DHS) program, which assists countries in periodically collecting data to monitor and evaluate populations and health and nutrition programs. The MDHS 2015-2016 was implemented by the National Statistical Office from October 19, 2015 to February 18, 2016. The survey involved interviews with a representative sample of women and men, and collected information on households, data on biomarkers, and anthropometric measurements of women (15-49 years of age) and children under 5 years of age, among other information. As part of this survey, 24,562 women aged 15-49 years were interviewed. We excluded 1,883 women who self-reported that they were pregnant, 3,022 who were below 18 years of age and 13,254 who had missing or flagged height and weight measurements, or for whom anthropometry was not done for other reasons (e.g., refusal). Pregnant women were excluded because pregnancy nullifies results for body mass index (BMI) as an indicator of nutrition status, especially at the population level, and women aged 15-17 years were excluded because they are still undergoing rapid physical changes, meaning that BMI alone is not a good indicator of their nutritional status (in this case overweight and obesity). The result was a final analytical sample of 6,443 adult non-pregnant women aged 18-49 years. Our study involved an exclusive population of women only, because anthropometric data for the MDHS was collected only in women. In addition, globally, more women than men are overweight or obese, and maternal overweight and obesity are associated with numerous health consequences and an emerging cycle of these conditions across multiple generations if left unchecked [11].

\section{Anthropometric measurements}

Height and weight were recoded following the Centers for Disease Control anthropometry protocol [12]. Height was measured in a standing position using standard height boards, while the participant was in a relaxed state. Shoulders were stress-free, and hair pins and all other head buns and ornaments were removed. Each woman was directed to stand straight, with her back against the board, toes apart, and heels together. Height was measured to the nearest $0.1 \mathrm{~cm}$. A calibrated digital scale was used to measure weight. The scale was put on a flat surface and tared to zero. The participant was requested to put on light clothing, and stand straight on the scale, with eyes focused in front. Weight was measured to the nearest $0.1 \mathrm{~kg}$. Height and weight were used to estimate the prevalence of overweight and obesity using BMI (calculated as a continuous variable using the following formula: weight $[\mathrm{kg}] / \mathrm{hei}$ ght $\left.[\mathrm{m}]^{2}\right)$. 


\section{Quantitative variables in the study}

The main outcome variable was overweight and obesity (BMI $\geq 25.0 \mathrm{~kg} / \mathrm{m}^{2}$ ). For the regression analysis, BMI was recoded into a binary variable with the categories of not overweight or obese $\left(\mathrm{BMI} \leq 24.9 \mathrm{~kg} / \mathrm{m}^{2}\right)$ and overweight or obese $\left(\geq 25.0 \mathrm{~kg} / \mathrm{m}^{2}\right)$. For other ancillary analyses, BMI was categorized based on the WHO [13] classes as follows: underweight $\left(<18.5 \mathrm{~kg} / \mathrm{m}^{2}\right)$, normal (18.5$\left.24.9 \mathrm{~kg} / \mathrm{m}^{2}\right)$, overweight $\left(25.0-29.9 \mathrm{~kg} / \mathrm{m}^{2}\right)$, obese $\left(\geq 30.0 \mathrm{~kg} / \mathrm{m}^{2}\right)$, pre-obese $\left(25.0-29.9 \mathrm{~kg} / \mathrm{m}^{2}\right)$, obese class I $\left(30.0-34.9 \mathrm{~kg} / \mathrm{m}^{2}\right)$, obese class II $\left(35.0-39.9 \mathrm{~kg} / \mathrm{m}^{2}\right)$, and obese class III $\left(\geq 40.0 \mathrm{~kg} / \mathrm{m}^{2}\right)$.

The covariates (social and demographic associates) were presented and defined as follows: age in years (calculated as a continuous variable and stratified into 6 categories: 18-22, 23-27, 28-32, $33-37,38-42$ and $\geq 43$ years), area of residence (rural or urban), marital status (recoded into 6 categories: never in union, married, living with partner, widowed, divorced, and separated), educational status (categorized as no education, primary education, secondary education, and higher education), occupation (recoded and categorized into 8 categories based on the International Standard Classification of Occupations [14]: not working, professional/ managerial/technician, clerical/sales, agriculture, household and domestic, services, skilled manual and unskilled manual), socioeconomic status (SES) (indicators of SES were assessed using the wealth index quintile, ranging from first to fifth, which translate to poorest to richest in that order), health insurance (yes or no regardless of the insurance source), parity (number of births in the previous 5 years, categorized as none, 1 , or $\geq 2$ births), total number of people living in the household ( 3 or fewer, 4-7, 8-10, or $\geq 11$ people), current smoking status (does not smoke or currently smokes), contraceptive use (using any form of contraceptive or not using any form of contraceptive, religion (recoded into 5 categories: none, other Christian, Muslim, Catholic, and Protestant), Ethnicity (recoded into 6 categories: others, Tumbuka, Lhomwe, Yao, Ngoni, and Chewa).

\section{Statistical analysis}

The analysis was carried out in SPSS version 20.0 (IBM Corp., Armonk, NY, USA) for descriptive statistics (frequencies and weighted percentages) and binary logistic regression to evaluate associations and determine odds. Frequency was analyzed and presented as unweighted, but percentages were weighted so that the results represent the Malawian adult non-pregnant women population. The prevalence estimates for underweight, normal weight, overweight, and obesity were computed as percentages with the total sample size as the denominator. Furthermore, the prevalence of overweight and obesity and their associations with different categorical background characteristics were analyzed using the chi-square test. Results were considered statistically significant at $p$-values $<0.05$. Binary logistic regression was used to determine possible relationships and the direction of the associations between different independent variables and the outcome. Bivariate binary logistic regression yielded crude odds ratios (with 95\% confidence intervals [CI]) for membership in the overweight or obese category. This was followed by multivariate logistic analysis to adjust for all the social and demographic determinants of the study (independent variables), thereby generating adjusted odds ratios (aOR) and 95\% CIs.

\section{Ethics statement}

Prior to conducting this research, a request for approval to download and use datasets (MDHS 2015-16) was sort from the DHS programme. The programme's institutional review board granted authorization (AuthLetter_120130). On the part of the data source, DHS maintains good ethical standards in all the surveys it conducts. The MDHS 2015-16 was preceded by a national campaign to raise public awareness of the survey aims and give information about its process. Participation in the survey conducted was completely voluntary and with full autonomy to take part or to reject participation at any point of the survey. No perceived potential harm was encountered on refusing participation. Informed, verbal individual consent was obtained from respondents before conducting the questionnaire or interview. All participants' information were processed anonymously and labeled with just Identification codes. The survey was supervised and approved by the Ministry of Health.

\section{RESULTS}

\section{Background characteristics}

The background characteristics of the study subjects are presented in Table 1. Of all the participants, $81.5 \%$ were from rural areas, $68.0 \%$ were married, and $59.0 \%$ had completed up to primary-level education. The 18-22 age group accounted for $23.7 \%$ of the participants, while the lowest percentage was found in the $38-42$ age group (10.9\%). With regard to religion, $26.4 \%$ of the respondents were Protestants, $17.5 \%$ Catholics, $12.6 \%$ Muslims, and over a quarter (42.9\%) belonged to other forms of Christianity. Furthermore, $23.6 \%$ of the subjects were in the fifth wealth index quintile, indicating the highest level of SES, $19.3 \%$ were in the poorest (first), 19.2\% were in the fourth quintile (rich), 19.1\% were poor (second quintile), and $18.8 \%$ were in the middle class (third quintile).

\section{Distribution of body mass index categories across subjects in Malawi}

Table 2 presents a summary of the anthropometric measurements of study subjects. The subjects in rural areas were marginally older than those in urban areas ( $30.00 \pm 8.70$ years vs. $29.00 \pm$ 7.80 years, respectively). The mean weight, height, and BMI of women were $56.31 \pm 10.40 \mathrm{~kg}, 1.56 \pm 0.06 \mathrm{~m}$, and $23.06 \pm 3.94 \mathrm{~kg} /$ $\mathrm{m}^{2}$, respectively. Differences in background characteristics were significantly associated with BMI, except for region of residence $(p<0.001$ vs. $p=0.056)$ (Table 3$)$. Overall, $5.7 \%$ of the women fell into the $<18.5 \mathrm{~kg} / \mathrm{m}^{2}$ (underweight) category of BMI, with no significant differences by area of residence $5.2 \%$ in urban areas vs. $5.8 \%$ in rural areas; $p=0.046$; comparison with an alpha of 
Table 1. Background characteristics of the study subjects $(n=6,443)$

\begin{tabular}{|c|c|}
\hline Characteristics & n (\%) \\
\hline \multicolumn{2}{|l|}{ Age (yr) } \\
\hline $18-22$ & $1,530(23.7)$ \\
\hline $23-27$ & $1,316(20.5)$ \\
\hline $28-32$ & $1,179(18.4)$ \\
\hline $33-37$ & 969 (15.0) \\
\hline $38-42$ & 711 (10.9) \\
\hline$\geq 43$ & 738 (11.5) \\
\hline \multicolumn{2}{|l|}{ Region } \\
\hline Northern & $1,230(11.3)$ \\
\hline Central & $2,201(42.5)$ \\
\hline Southern & $3,012(46.2)$ \\
\hline \multicolumn{2}{|l|}{ Residence } \\
\hline Urban & $1,401(18.5)$ \\
\hline Rural & $5,042(81.5)$ \\
\hline \multicolumn{2}{|l|}{ Marital status } \\
\hline Never in union/single & 783 (11.6) \\
\hline Married & $4,318(68.0)$ \\
\hline Living with partner & $324(4.7)$ \\
\hline Widowed & $239(3.5)$ \\
\hline Divorced & $380(6.1)$ \\
\hline Separated & $399(6.0)$ \\
\hline \multicolumn{2}{|l|}{ Education } \\
\hline None & $856(13.8)$ \\
\hline Primary & $3,726(59.0)$ \\
\hline Secondary & $1,655(23.6)$ \\
\hline Higher & $206(3.6)$ \\
\hline \multicolumn{2}{|l|}{ Religion } \\
\hline Catholic & $1,110(17.5)$ \\
\hline Protestant & $1,817(26.4)$ \\
\hline Other Christian & $2,766(42.9)$ \\
\hline Muslim & 722 (12.6) \\
\hline None & $28(0.5)$ \\
\hline \multicolumn{2}{|l|}{ Ethnicity } \\
\hline Chewa & $1,922(34.1)$ \\
\hline Tumbuka & $658(8.9)$ \\
\hline Lhomwe & 1,197 (19.8) \\
\hline Yao & 747 (13.7) \\
\hline Ngoni & $813(11.8)$ \\
\hline Others & $1,106(11.8)$ \\
\hline \multicolumn{2}{|l|}{ Occupation } \\
\hline Professional/technician/managerial & $392(5.5)$ \\
\hline Clerical/sales & $408(6.2)$ \\
\hline Agriculture (self/employee) & $2,574(41.3)$ \\
\hline Household and domestic & $92(1.8)$ \\
\hline Services & $70(1.4)$ \\
\hline Skilled manual & $113(1.7)$ \\
\hline Unskilled manual & $911(14.1)$ \\
\hline Not working & $1,883(28.1)$ \\
\hline
\end{tabular}

(Continued to the next)
Table 1. Continued

\begin{tabular}{|c|c|}
\hline Characteristics & $\mathrm{n}(\%)$ \\
\hline \multicolumn{2}{|l|}{ Parity in previous $5 \mathrm{yr}$} \\
\hline No & $2,395(36.7)$ \\
\hline 1 & $2,887(45.1)$ \\
\hline$\geq 2$ & $1,161(18.2)$ \\
\hline \multicolumn{2}{|l|}{ Covered by health insurance } \\
\hline No & $6,343(98.3)$ \\
\hline Yes & $100(1.7)$ \\
\hline \multicolumn{2}{|l|}{ Contraceptive use (of any form) } \\
\hline Not using & $2,861(44.2)$ \\
\hline Using & $3,528(55.8)$ \\
\hline \multicolumn{2}{|l|}{ Self-reported menopause status } \\
\hline Not in menopause & $6,262(97.2)$ \\
\hline In menopause & $181(2.8)$ \\
\hline \multicolumn{2}{|l|}{ Current smoking status } \\
\hline Does not smoke & $6,392(99.3)$ \\
\hline Smokes & $51(0.7)$ \\
\hline \multicolumn{2}{|c|}{ Who makes decision concerning respondent's health care } \\
\hline Others & $5(0.1)$ \\
\hline Respondent alone & $890(18.9)$ \\
\hline Respondent and husband/partner & $2,375(50.5)$ \\
\hline Husband/partner alone & $1,351(30.1)$ \\
\hline Someone else & $21(0.4)$ \\
\hline \multicolumn{2}{|l|}{ SES quintiles } \\
\hline First (poorest) & $1,097(19.3)$ \\
\hline Second & $1,195(19.1)$ \\
\hline Third & $1,186(18.8)$ \\
\hline Fourth & $1,282(19.2)$ \\
\hline Fifth (richest) & $1,683(23.6)$ \\
\hline
\end{tabular}

SES, socioeconomic status.

Table 2. Age, weight, height, and BMl of subjects by area of residence

\begin{tabular}{lclcc}
\hline Areas & Age $(\mathrm{yr})$ & Weight $(\mathrm{kg})$ & Height $(\mathrm{m})$ & $\mathrm{BMI}\left(\mathrm{kg} / \mathrm{m}^{2}\right)$ \\
\hline Urban & $29.00 \pm 7.80$ & $61.43 \pm 13.01$ & $1.57 \pm 0.06$ & $24.80 \pm 4.91$ \\
Rural & $30.00 \pm 8.70$ & $55.12 \pm 9.35$ & $1.56 \pm 0.06$ & $22.66 \pm 3.60$ \\
$\begin{array}{l}\text { Residence } \\
\text { combined }\end{array}$ & $30.22 \pm 8.60$ & $56.31 \pm 10.40$ & $1.56 \pm 0.06$ & $23.06 \pm 3.94$ \\
\hline
\end{tabular}

Values are presented as mean \pm standard deviation.

$\mathrm{BMI}$, body mass index.

0.006) (Table 3). Approximately $16.8 \%$ of the respondents fell into the BMI category of $25.0-29.9 \mathrm{~kg} / \mathrm{m}^{2}$ (pre-obese/overweight). The distribution of overweight was significantly different between urban and rural residents (25.5\% in urban areas and $14.8 \%$ in rural areas; $\mathrm{p}<0.001)$. Overweight was almost equally pronounced in women aged $28-32$ years and those aged $33-37$ years $(21.6 \%$ and $21.4 \%$, respectively). Over a quarter $(26.1 \%)$ of women with a higher level of education and those in the richest wealth quintile (25.8\%) were overweight. Overall, $6.3 \%$ of women were in the 
Table 3. Distribution of BMI categories among subjects in Malawi

\begin{tabular}{|c|c|c|c|c|c|}
\hline & \multicolumn{5}{|c|}{$\mathrm{BMI}\left(\mathrm{kg} / \mathrm{m}^{2}\right)$} \\
\hline & $\begin{array}{c}<18.50 \\
\text { (under- } \\
\text { weight) }\end{array}$ & $\begin{array}{l}18.50- \\
24.99 \\
\text { (normal } \\
\text { weight) }\end{array}$ & $\begin{array}{c}25.00- \\
29.99 \\
\text { (over- } \\
\text { weight) }\end{array}$ & $\begin{array}{l}\geq 30.00 \\
\text { (obese) }\end{array}$ & $p$-value ${ }^{1}$ \\
\hline Age (yr) & & & & & $<0.001$ \\
\hline $18-22$ & 6.3 & 82.2 & 10.6 & 0.9 & \\
\hline $23-27$ & 4.7 & 76.8 & 13.8 & 4.7 & \\
\hline $28-32$ & 5.7 & 65.0 & 21.6 & 7.7 & \\
\hline $33-37$ & 5.6 & 61.3 & 21.4 & 11.6 & \\
\hline $38-42$ & 6.2 & 67.2 & 19.0 & 7.6 & \\
\hline$\geq 43$ & 5.8 & 65.3 & 19.0 & 9.9 & \\
\hline Region & & & & & 0.056 \\
\hline Northern & 5.3 & 67.9 & 20.3 & 6.5 & \\
\hline Central & 5.4 & 72.0 & 15.8 & 6.8 & \\
\hline Southern & 6.1 & 71.3 & 16.8 & 5.8 & \\
\hline Residence & & & & & $<0.001$ \\
\hline Urban & 5.2 & 54.7 & 25.5 & 14.6 & \\
\hline Rural & 5.8 & 75.0 & 14.8 & 4.4 & \\
\hline$p$-value ${ }^{2}$ & 0.046 & & $<0.001$ & $<0.001$ & \\
\hline Marital status & & & & & $<0.001$ \\
\hline Never in union & 7.8 & 73.9 & 15.4 & 3.0 & \\
\hline Married & 5.2 & 70.6 & 16.9 & 7.4 & \\
\hline Living with partner & 4.3 & 76.2 & 17.2 & 2.3 & \\
\hline Widowed & 6.3 & 64.4 & 20.7 & 8.6 & \\
\hline Divorced & 7.7 & 68.3 & 19.6 & 4.4 & \\
\hline Separated & 6.3 & 76.6 & 13 & 4.2 & \\
\hline Education & & & & & $<0.001$ \\
\hline None & 6.5 & 70.1 & 18.3 & 5.1 & \\
\hline Primary & 5.5 & 75.0 & 14.6 & 4.8 & \\
\hline Secondary & 6.2 & 64.7 & 19.9 & 9.2 & \\
\hline Higher & 1.7 & 57.0 & 26.1 & 15.2 & \\
\hline Religion & & & & & $<0.001$ \\
\hline None & 12.5 & 62.5 & 15.6 & 9.4 & \\
\hline Catholic & 5.4 & 71.1 & 18.6 & 4.9 & \\
\hline Protestant & 3.9 & 69.8 & 19.4 & 6.9 & \\
\hline Other Christian & 6.4 & 71.9 & 15.3 & 6.4 & \\
\hline Muslim & 7.2 & 72.5 & 13.8 & 6.6 & \\
\hline Ethnicity & & & & & $<0.001$ \\
\hline Others & 5.2 & 69.5 & 17.3 & 8.1 & \\
\hline Chewa & 5.6 & 74.4 & 15.1 & 4.8 & \\
\hline Tumbuka & 4.2 & 68.4 & 20.3 & 7.1 & \\
\hline Lhomwe & 6.3 & 72.0 & 15.9 & 5.7 & \\
\hline Yao & 6.1 & 71.1 & 14.9 & 7.9 & \\
\hline Ngoni & 5.8 & 64.6 & 22.1 & 7.4 & \\
\hline Occupation & & & & & $<0.001$ \\
\hline Not working & 5.9 & 71.0 & 17.7 & 5.5 & \\
\hline $\begin{array}{c}\text { Professional/mana- } \\
\text { gerial/technician }\end{array}$ & 3.7 & 53.3 & 27.2 & 15.9 & \\
\hline Clerical/sales & 4.3 & 63.5 & 20.5 & 11.6 & \\
\hline $\begin{array}{l}\text { Agriculture (self/ } \\
\text { employee) }\end{array}$ & 5.7 & 78 & 12.9 & 3.4 & \\
\hline
\end{tabular}

(Continued to the next)
Table 3. Continued

\begin{tabular}{|c|c|c|c|c|c|}
\hline & \multicolumn{5}{|c|}{ BMI $\left(\mathrm{kg} / \mathrm{m}^{2}\right)$} \\
\hline & $\begin{array}{l}<18.50 \\
\text { (under- } \\
\text { weight) }\end{array}$ & $\begin{array}{c}18.50- \\
24.99 \\
\text { (normal } \\
\text { weight) }\end{array}$ & $\begin{array}{c}25.00- \\
29.99 \\
\text { (over- } \\
\text { weight) }\end{array}$ & $\begin{array}{l}\geq 30.00 \\
\text { (obese) }\end{array}$ & $p$-value ${ }^{1}$ \\
\hline $\begin{array}{l}\text { Household and } \\
\text { domestic }\end{array}$ & 0.9 & 65.5 & 26.5 & 7.1 & \\
\hline Services & 8.0 & 55.7 & 29.5 & 6.8 & \\
\hline Skilled manual & 3.7 & 57.0 & 27.1 & 12.1 & \\
\hline Unskilled manual & 7.2 & 66.1 & 17.0 & 9.6 & \\
\hline SES quintile & & & & & $<0.001$ \\
\hline First (poorest) & 7.1 & 80.1 & 10.9 & 1.9 & \\
\hline Second & 5.2 & 80.8 & 10.8 & 3.3 & \\
\hline Third & 5.2 & 75.7 & 15.5 & 3.6 & \\
\hline Fourth & 6.2 & 68.7 & 18.8 & 6.3 & \\
\hline Fifth (richest) & 4.9 & 54.7 & 25.8 & 14.6 & \\
\hline
\end{tabular}

Values are presented as \%.

BMI, body mass index; SES, socioeconomic status.

'Pearson chi-square test.

${ }^{2}$ Met the assumption for Bonferroni correction for within-column probabilities (alpha=0.006): multiple-wise analysis is limited, hence not inflating the 'family' (type I) error [15].

obese (BMI $\geq 30.0 \mathrm{~kg} / \mathrm{m}^{2}$ ) category, with significant differences between rural and urban areas (14.6\% in urban areas vs. $4.4 \%$ in rural areas; $\mathrm{p}<0.001)$. Obesity was more pronounced in women aged 33-37 years (11.6\%), those with a higher level of education (15.2\%), those who had a professional/managerial/technician occupation (15.9\%), and those in the richest SES group (14.6\%).

Overall, the prevalence of overweight and obesity among adult women in the study was $16.8 \%$ and $6.3 \%$, respectively. In urban areas, $25.5 \%$ and $14.6 \%$ of the women were overweight and obese, respectively. In contrast, in rural areas, $14.8 \%$ and $4.4 \%$ of the women were overweight and obese, respectively.

An ancillary categorization of obesity (BMI $\geq 30.0 \mathrm{~kg} / \mathrm{m}^{2}$ ) (Figure 1) by area of residence showed that $4.9 \%$ of the respondents were obese class I, $1.0 \%$ were obese class II, and $0.3 \%$ were obese class III. In urban areas, $11.0 \%, 2.5 \%$, and $1.2 \%$ of the respondents were obese class I, obese class II, and obese class III, respectively. In rural areas, 3.6\% of the respondents were obese class I, $0.7 \%$ were obese class II, and $0.1 \%$ were obese class III. The distribution of pre-obese, obese class I, obese class II, and obese class III women was also considered in reference to SES strata (Table 4). Approximately $11.3 \%$ of women in the highest SES stratum were obese class I, and more than a quarter (25.8\%) were pre-obese. In the poorest stratum, the prevalence of pre-obese women was $10.9 \%$, marginally higher than in poor women (10.8\%).

\section{Social determinants associated with overweight and obesity among study subjects in Malawi}

Table 5 shows the results of binary logistic regression for the predictive probability for membership in the category of overwei- 


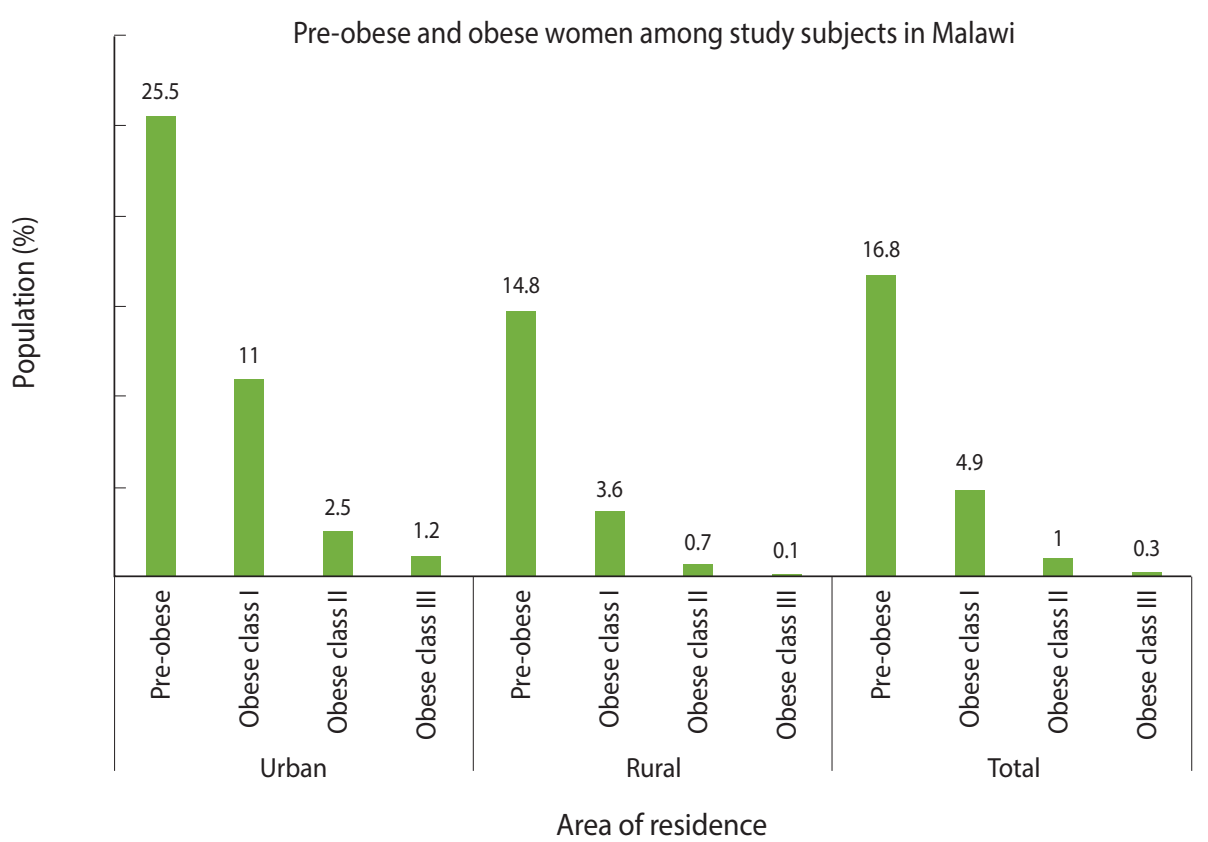

Figure 1. Presents a summary of the prevalence of overweight and obesity by area of residence.

Table 4. Pre-obese/overweight, class I, class II, and class III obese women across socioeconomic status (SES) strata in Malawi

\begin{tabular}{|c|c|c|c|c|c|}
\hline \multirow[b]{2}{*}{ SES quintiles } & \multicolumn{5}{|c|}{ Body mass index $\left(\mathrm{kg} / \mathrm{m}^{2}\right)$} \\
\hline & $\begin{array}{c}\text { Pre-obese } \\
(25.00-29.99)\end{array}$ & $\begin{array}{l}\text { Obese class I } \\
(30.00-34.99)\end{array}$ & $\begin{array}{l}\text { Obese class II } \\
(35.00-39.99)\end{array}$ & $\begin{array}{l}\text { Obese class III } \\
\qquad(\geq 40.00)\end{array}$ & $\mathrm{p}$-value ${ }^{1}$ \\
\hline First (poorest) & $106(10.9)$ & $13(1.3)$ & $5(0.5)$ & - & $<0.001$ \\
\hline Second & $136(10.8)$ & $30(3.0)$ & $3(0.2)$ & $1(0.1)$ & \\
\hline Third & $186(15.5)$ & $32(2.7)$ & $11(0.8)$ & - & \\
\hline Fourth & $255(18.8)$ & $62(5.0)$ & $11(0.9)$ & $6(0.4)$ & \\
\hline Fifth (richest) & $439(25.8)$ & $201(11.3)$ & $39(2.4)$ & $21(0.9)$ & \\
\hline Total & $1,122(16.8)$ & 338 (4.9) & $69(1.0)$ & $28(0.3)$ & \\
\hline
\end{tabular}

Values are presented as frequency number (\%).

'Pearson chi-square test.

ght or obese. Women who were in the age group of 33-37 years were 4 times more likely to be overweight or obese than those aged between 18 and 22 years (aOR, 3.95; 95\% CI, 2.91 to 5.36; $\mathrm{p}<0.001)$. The odds of a woman residing in urban area being overweight or obese were $18.0 \%$ higher than those of a woman residing in rural area (aOR, 1.18; 95\% CI, 0.93 to 1.49). Married women were less likely to be overweight or obese than those who had never been married. The crude odds ratios suggested that the odds of being overweight or obese increased with increasing levels of education, but this was not confirmed in the multivariate analysis. Women from the Ngoni ethnic group were more likely to be overweight or obese than women of other ethnic groups in Malawi (aOR, 1.54; 95\% CI, 1.14 to 2.08 ; $\mathrm{p}<0.05$ ).

Women in agricultural occupations (self-employed or employee) were less likely to be overweight or obese, which was highly statistically significant (aOR, $0.69 ; 95 \% \mathrm{CI}, 0.57$ to $0.84 ; \mathrm{p}<0.001$ ), while women who were skilled manual workers were more likely to be overweight or obese than those who were not working (aOR, 1.59; $95 \%$ CI, 0.96 to 2.64). The risk of being overweight or obese increased with increasing SES, as women belonging to the richest (fifth quintile of the wealth index) group were over 3 times more likely to be overweight or obese than those in the poorest quintile (aOR, 3.30; 95\% CI, 2.46 to 4.43). Coverage by any form of health insurance was associated with being overweight or obese, and current smokers were less likely to be overweight or obese. A higher likelihood of being overweight or obese was seen in women residing in households with 8-10 people, as compared to those having 3 or fewer people (aOR, 1.09; 95\% CI, 0.81 to 1.46 ).

\section{DISCUSSION}

Using the MDHS 2015-2016 data, we assessed the overall distribution of BMI (focusing on overweight and obesity) across different social and demographic categories. Furthermore, we deter- 
Table 5. Logistic regression for determinants of overweight/obesity among women in Malawi

\begin{tabular}{|c|c|c|}
\hline Variables & $\begin{array}{l}\text { Crude OR } \\
(95 \% \mathrm{Cl})\end{array}$ & $\begin{array}{l}\text { Adjusted OR } \\
(95 \% \mathrm{CI})\end{array}$ \\
\hline \multicolumn{3}{|l|}{ Age (yr) } \\
\hline $18-22$ & 1.00 (reference) & 1.00 (reference) \\
\hline $23-27$ & $1.74(1.41,2.14)$ & $1.53(1.14,2.06)$ \\
\hline $28-32$ & $3.17(2.59,3.88)$ & $3.06(2.28,4.10)$ \\
\hline $33-37$ & $3.77(3.06,4.63)$ & $3.95(2.91,5.36)$ \\
\hline $38-42$ & $2.76(2.20,3.48)$ & $3.02(2.15,4.24)$ \\
\hline$\geq 43$ & $3.10(2.48,3.88)$ & $3.26(2.29,4.64)$ \\
\hline \multicolumn{3}{|l|}{ Region } \\
\hline Northern & 1.00 (reference) & 1.00 (reference) \\
\hline Central & $0.80(0.66,0.97)$ & $0.89(0.65,1.21)$ \\
\hline Southern & $0.79(0.66,0.97)$ & $0.82(0.60,1.12)$ \\
\hline \multicolumn{3}{|l|}{ Residence } \\
\hline Rural & 1.00 (reference) & 1.00 (reference) \\
\hline Urban & $2.82(2.47,3.23)$ & $1.18(0.93,1.49)$ \\
\hline \multicolumn{3}{|l|}{ Marital Status } \\
\hline Never in union/single & 1.00 (reference) & 1.00 (reference) \\
\hline Married & $1.43(1.17,1.74)$ & $0.93(0.68,1.27)$ \\
\hline Living with partner & $1.07(0.76,1.50)$ & - \\
\hline Widowed & $1.86(1.32,2.63)$ & - \\
\hline Divorced & $1.42(1.05,1.91)$ & - \\
\hline Separated & $0.94(0.68,1.29)$ & - \\
\hline \multicolumn{3}{|l|}{ Education } \\
\hline None & 1.00 (reference) & 1.00 (reference) \\
\hline Primary & $0.79(0.66,0.94)$ & $0.77(0.62,0.96)$ \\
\hline Secondary & $1.34(1.11,1.63)$ & $0.91(0.68,1.21)$ \\
\hline Higher & $2.30(1.69,3.11)$ & $0.97(0.58,1.64)$ \\
\hline \multicolumn{3}{|l|}{ Religion } \\
\hline None & 1.00 (reference) & 1.00 (reference) \\
\hline Other Christian & $0.79(0.36,1.75)$ & $0.84(0.32,2.17)$ \\
\hline Muslim & $0.72(0.32,1.64)$ & $0.81(0.30,2.21)$ \\
\hline Catholic & $0.87(0.39,1.95)$ & $0.81(0.31,2.13)$ \\
\hline Protestant & $1.02(0.46,2.26)$ & $0.97(0.37,2.55)$ \\
\hline \multicolumn{3}{|l|}{ Ethnicity } \\
\hline Others & 1.00 (reference) & 1.00 (reference) \\
\hline Tumbuka & $1.11(0.87,1.43)$ & $0.99(0.69,1.40)$ \\
\hline Lhomwe & $0.81(0.66,1.01)$ & $1.10(0.83,1.47)$ \\
\hline Yao & $0.87(0.69,1.09)$ & $1.37(0.95,1.98)$ \\
\hline Ngoni & $1.24(0.99,1.56)$ & $1.54(1.14,2.08)$ \\
\hline Chewa & $0.73(0.60,0.89)$ & $1.08(0.81,1.44)$ \\
\hline \multicolumn{3}{|l|}{ Occupation } \\
\hline Not working & 1.00 (reference) & 1.00 (reference) \\
\hline $\begin{array}{l}\text { Professional/technician/ } \\
\text { managerial }\end{array}$ & $2.49(1.97,3.16)$ & $0.99(0.71,1.39)$ \\
\hline Clerical/sales & $1.56(1.23,1.99)$ & $1.01(0.74,1.37)$ \\
\hline Agriculture (self/employee) & $0.65(0.56,0.75)$ & $0.69(0.57,0.84)$ \\
\hline Household and domestic & $1.68(1.12,2.52)$ & $1.28(0.63,2.60)$ \\
\hline Services & $1.85(1.18,2.90)$ & $1.22(0.64,2.34)$ \\
\hline Skilled manual & $2.10(1.40,3.15)$ & $1.59(0.96,2.64)$ \\
\hline Unskilled manual & $1.21(1.00,1.45)$ & $1.07(0.85,1.36)$ \\
\hline
\end{tabular}

Table 5. Continued

\begin{tabular}{|c|c|c|}
\hline Variables & $\begin{array}{c}\text { Crude OR } \\
(95 \% \mathrm{Cl})\end{array}$ & $\begin{array}{l}\text { Adjusted OR } \\
\qquad(95 \% \mathrm{Cl})\end{array}$ \\
\hline \multicolumn{3}{|l|}{ Parity in previous $5 \mathrm{yr}$} \\
\hline No & 1.00 (reference) & 1.00 (reference) \\
\hline 1 & $0.73(0.64,0.83)$ & $0.95(0.79,1.15)$ \\
\hline$\geq 2$ & $0.47(0.39,0.56)$ & $0.72(0.56,0.93)$ \\
\hline \multicolumn{3}{|l|}{ Covered by health insurance } \\
\hline No & 1.00 (reference) & 1.00 (reference) \\
\hline Yes & $2.48(1.68,3.65)$ & $1.28(0.71,2.29)$ \\
\hline \multicolumn{3}{|l|}{ Contraceptive use (of any form) } \\
\hline No & 1.00 (reference) & 1.00 (reference) \\
\hline Yes & $0.91(0.81,1.03)$ & $0.95(0.81,1.11)$ \\
\hline \multicolumn{3}{|l|}{ Self-reported menopause status } \\
\hline Not in menopause & 1.00 (reference) & 1.00 (reference) \\
\hline In menopause & $0.88(0.61,1.27)$ & $0.75(0.48,1.17)$ \\
\hline \multicolumn{3}{|l|}{ Current smoking status } \\
\hline Not smoking & 1.00 (reference) & 1.00 (reference) \\
\hline Smoking & $0.82(0.39,1.72)$ & $0.92(0.41,2.09)$ \\
\hline \multicolumn{3}{|c|}{ Who makes decision concerning respondent's health care } \\
\hline Others & 1.00 (reference) & 1.00 (reference) \\
\hline Respondent alone & $0.62(0.09,3.99)$ & $0.33(0.05,2.33)$ \\
\hline $\begin{array}{l}\text { Respondent and husband/ } \\
\text { partner }\end{array}$ & $0.68(0.11,4.29)$ & $0.37(0.05,2.63)$ \\
\hline Respondent and other person & $0.49(0.08,3.09)$ & $0.32(0.05,2.23)$ \\
\hline Husband/partner alone & $0.77(0.09,6.32)$ & $0.63(0.07,5.98)$ \\
\hline \multicolumn{3}{|l|}{ Total household members ( $\mathrm{n}$ ) } \\
\hline$\leq 3$ & 1.00 (reference) & 1.00 (reference) \\
\hline $4-7$ & $1.03(0.89,1.19)$ & $0.82(0.66,1.02)$ \\
\hline $8-10$ & $1.23(0.99,1.51)$ & $1.09(0.81,1.46)$ \\
\hline$\geq 11$ & $1.12(0.71,1.77)$ & $0.91(0.48,1.71)$ \\
\hline \multicolumn{3}{|l|}{ SES quintiles } \\
\hline First (poorest) & 1.00 (reference) & 1.00 (reference) \\
\hline Second & $1.12(0.89,1.42)$ & $1.08(0.82,1.43)$ \\
\hline Third & $1.61(1.29,2.01)$ & $1.31(1.00,1.71)$ \\
\hline Fourth & $2.29(1.85,2.83)$ & $1.67(1.28,2.18)$ \\
\hline Fifth (richest) & $4.64(3.81,5.65)$ & $3.30(2.46,4.43)$ \\
\hline
\end{tabular}

For the adjusted ORs, multivariate logistic regression was done, adjusting for all other covariates to address possible confounding; For crude ORs, univariate logistic regression was done.

ORs, odd ratios; $\mathrm{Cl}$, confidence interval; SES, socioeconomic status.

mined the different social determinants of overweight and obesity in adult non-pregnant women in Malawi. Different social factors showed significant correlations with overweight and obesity. Overall, the study found that there was a relatively low prevalence of underweight and a relatively high prevalence of overweight and obesity. The combined prevalence of overweight and obesity in adult non-pregnant women of Malawi was estimated to be $23.1 \%$. The results found are comparable with those found in Nigeria (29.2\%) [16], but are in contrast with those found in Uganda (11.3\%) [17]. The prevalence of overweight and obesity varied across different background characteristics of the women in Malawi. This dispari- 
ty has also been observed in Nepal [18].

The prevalence of overweight and obesity was higher in urban areas than in rural areas. A high burden of overweight and obesity in women residing in urban areas was also reported in other LMICs, including African countries [19-21]. This tendency could be attributed to rural residents being more actively involved in a less sedentary lifestyle and more laborious activities [22] than urban residents, whose occupations may encourage sedentariness. It should be noted that the high prevalence of overweight and obesity in women may also be due to their physiology, as they tend to deposit more fat than lean mass [23-25].

One of the novel elements of this study is that it further categorized BMIs of $\geq 25.0 \mathrm{~kg} / \mathrm{m}^{2}$ into categories of pre-obese, obese class I, obese class II, and obese class III, in order to elucidate clearly the severity of the problem and the need for an immediate response in terms of interventions. Despite the fact that poverty is an established factor in Malawi, there were still some women who were obese class III; this confirms that even the most severe form of obesity is not only a problem of the affluent. Obesity is a morbid state that requires immediate reversal to prevent premature mortality [26].

This study found that SES and age were highly significant determinants of overweight and obesity among women in Malawi. Higher levels of SES were associated with an increased prevalence and likelihood of being overweight or obese in this study, indicating that these women could be susceptible to NCDs associated with being overweight or obese. These findings could be explained by the availability of affordable, energy-dense foods due to urban sprawl, as well as reduced physical activity resulting in a sedentary lifestyle, as observed in a study in Kenya where the women who were most sedentary were in the highest income group [27], and in other studies finding that high-income households purchased foods in bulk and were more likely to overconsume the food [28]. These findings differ from those reported in high-income countries, people with a lower SES were more likely to be overweight or obese due to their tendency to purchase low-quality foods [29]. Multiple studies have indicated that overweight and obesity in women tend to increase with age [30,31]. Similarly, in our study, the odds of being overweight or obese from the ages of 30 years and above were almost 4 times as high compared to women in the 18-22-year-old age category. Starting at the age of 30 years, anecdotal evidence in developing countries shows that different family transitions happen, and women tend to shift various household roles to their children, making themselves more sedentary. In addition, studies have shown that as some women grow old, they tend to express less willingness to reduce weight irrespective of their health status [32]. In resource-restricted healthcare systems like that of Malawi, interventions on curbing overweight and obesity can therefore be prioritized for such age groups.

With regard to area of residence, women who were residing in urban areas were more likely to be overweight or obese. An urban environment is usually associated with an improved economic status of households; households with a high income tend to purchase food in bulk, spending more on both healthy and less healthy foods [32]. This study had some unique key findings. For example, in Malawi, women who belonged to the Ngoni ethnic group were found to have a $54.0 \%$ higher risk of being overweight or obese as compared to others (aOR, 1.54; 95\% CI, 1.14 to 2.08 ; $\mathrm{p}<0.05$ ). Evidence shows that the Ngoni people live a life of hefty meat consumption and alcohol drinking, and chiefly perceive themselves as living a 'healthy satisfactory life' [33]. The Ngoni people of Malawi are originally from South Africa and share their roots with the Zulu people, who in contrast to the public health notion that overweight and obesity are unhealthy-view plump women as "healthy" [34].

This study had some limitations. It was cross-sectional in nature and therefore cannot be used to infer causality. However, the motivation was to describe the distribution of overweight and obesity and provide clues regarding potential associations that can be further explored using robust study designs. Despite the essential limitations of BMI as a measure of weight status, it remains the most widely used measure for assessing weight status at the population level. We recognize the importance of measuring proximate factors for overweight and obesity such as physical activity and dietary intake; however, this was beyond the scope of this study and the relevant information was not available in the MDHS data.

Despite its limitations, the main strength of this study is its large sample size and the random clustering sampling method used to recruit participants in the survey, which made it a good representation of adult non-pregnant women in Malawi. The quality of the data is assured as the DHS uses well-trained field personnel, a standardized protocol, and validated tools in the data collection process. The inclusion criteria employed in this study were demanding and further strengthened the quality of the findings. This study also employed an analytical plan that stratified the results based on background characteristics, and also carefully controlled for confounding through a multivariate analysis. These results, therefore, present disaggregated information that is generalizable.

In conclusion, this study showed a high prevalence of overweight and obesity among adult non-pregnant women in Malawi. Overweight and obesity were closely associated with different social and demographic determinants, with factors such as age and SES increasing the risk of developing overweight and obesity by about 4 times in women. This study affirms that the roles of social determinants of overweight and obesity need to be mapped for better evidence-based prioritization of interventions and synergistic efforts to curb the epidemic. The time has come to prevent and control overweight and obesity.

\section{CONFLICT OF INTEREST}

The authors have no conflicts of interest to declare for this study.

\section{ACKNOWLEDGEMENTS}

None. 


\section{AUTHOR CONTRIBUTIONS}

Conceptualization: LM, AK. Data curation: LM. Formal analysis: LM. Funding acquisition: None. Project administration: AK. Writing - original draft: LM. Writing - review \& editing: LM, AK.

\section{ORCID}

Leonard Mndala: https://orcid.org/0000-0003-0488-6972; Abhay Kudale: https://orcid.org/0000-0003-2887-1636

\section{REFERENCES}

1. World Health Organization. Overweight and obesity; 2018 [cited 2019 May 14]. Available from: https://www.who.int/news-room/ fact-sheets/detail/obesity-and-overweight.

2. Barakat-Haddad C, Saeed U, Elliott S. A longitudinal cohort study examining determinants of overweight and obesity in adulthood. Can J Public Health 2017;108:e27-e35.

3. Omran AR. The epidemiologic transition. A theory of the epidemiology of population change. Milbank Mem Fund Q 1971;49: 509-538.

4. Popkin BM, Adair LS, Ng SW. Global nutrition transition and the pandemic of obesity in developing countries. Nutr Rev 2012;70: 3-21.

5. World Health Organization. Global status report on non-communicable diseases 2014; 2015 [cited 2019 Oct 14]. Available from: https://apps.who.int/iris/bitstream/handle/10665/148114/978924 1564854_eng.pdf? sequence $=1$.

6. Dee A, Kearns K, O’Neill C, Sharp L, Staines A, O’Dwyer V, et al. The direct and indirect costs of both overweight and obesity: a systematic review. BMC Res Notes 2014;7:242.

7. Agyemang C, Boatemaa S, Frempong GA, Aikins A. Obesity in sub-Saharan Africa. In: Ahima RS, editor. Metabolic syndrome. Switzerland: Springer International Publishing; 2016, p. 1-13.

8. Steyn NP, Ne JH, Parker WA, Ayah R, Mbithe D. Dietary, social, and environmental determinants of obesity in Kenyan women. Scand J Public Health 2011;39:88-97.

9. Micklesfield LK, Lambert EV, Hume DJ, Chantler S, Pienaar PR, Dickie K, et al. Socio-cultural, environmental and behavioural determinants of obesity in black South African women. Cardiovasc J Afr 2013;24:369-375.

10. World Health Organization. A conceptual framework for action on the social determinants of health: debates, policy \& practice, case studies; 2010 [cited 2019 May 14]. Available from http:// apps.who.int/iris/bitstream/10665/44489/1/9789241500852_eng. pdf.

11. Meehan S, Beck CR, Mair-Jenkins J, Leonardi-Bee J, Puleston R. Maternal obesity and infant mortality: a meta-analysis. Pediatrics 2014; 133:863-871.

12. Centers for Disease Control and Prevention. National health and nutrition examination survey (NHANES); 2007 [cited 2019 Jun 1]. Available from https://www.cdc.gov/nchs/data/nhanes/nhanes_ 07_08/manual_an.pdf.

13. World Health Organization. Obesity: preventing and managing the global epidemic; 2000 [cited 2019 Oct 14]. Available from: https://www.who.int/nutrition/publications/obesity/WHO_TRS_894/ en/.

14. International Labour Office. International standard classification of occupations: structure, group definitions and correspondence tables; 2016 [cited 2019 May 21]. Available from https://www.ilo. org/wcmsp5/groups/public/@dgreports/@dcomm/@publ/documents/publication/wcms_172572.pdf.

15. Nakagawa S. A farewell to Bonferroni: the problems of low statistical power and publication bias. Behav Ecol 2004;15:1044-1045.

16. Adebayo RA, Balogun MO, Adedoyin RA, Obashoro-John OA, Bisiriyu LA, Abiodun OO. Prevalence and pattern of overweight and obesity in three rural communities in southwest Nigeria. Diabetes Metab Syndr Obes 2014;7:153-158.

17. Maher D, Waswa L, Baisley K, Karabarinde A, Unwin N, Grosskurth H. Distribution of hyperglycaemia and related cardiovascular disease risk factors in low-income countries: a cross-sectional population-based survey in rural Uganda. Int J Epidemiol 2011;40:160-171.

18. Al Kibria GM. Prevalence and factors affecting underweight, overweight and obesity using Asian and World Health Organization cutoffs among adults in Nepal: analysis of the Demographic and Health Survey 2016. Obes Res Clin Pract 2019;13:129-136.

19. Subramanian SV, Perkins JM, Özaltin E, Davey Smith G. Weight of nations: a socioeconomic analysis of women in low- to middle-income countries. Am J Clin Nutr 2011;93:413-421.

20. Atek M, Traissac P, El Ati J, Laid Y, Aounallah-Skhiri H, EymardDuvernay S, et al. Obesity and association with area of residence, gender and socio-economic factors in Algerian and Tunisian adults. PLoS One 2013;8:e75640.

21. Letamo G. The prevalence of, and factors associated with, overweight and obesity in Botswana. J Biosoc Sci 2011;43:75-84.

22. Sola AO, Steven AO, Kayode JA, Olayinka AO. Underweight, overweight and obesity in adults Nigerians living in rural and urban communities of Benue State. Ann Afr Med 2011;10:139-143.

23. Amegah AK, Lumor S, Vidogo F. Prevalence and determinants of overweight and obesity in adult residents of Cape Coast, Ghana: a hospital-based study. Afr J Food Agric Nutr Dev 2011;11: 4828-4846.

24. Mogre V, Mwinlenaa PP, Oladele J, Amalba A. Impact of physical activity levels and diet on central obesity among civil servants in Tamale metropolis. J Med Biomed Sci 2012;1:1-9.

25. Pobee RA, Owusu WB, Plahar WA. The prevalence of obesity among female teachers of child-bearing age in Ghana. Afr J Food Agric Nutr Dev 2013;13:7820-7839.

26. Dobbs R, Sawers C, Thompson F, Manyika J, Woetzel JR, Child P, et al. Overcoming obesity: an initial economic analysis. Executive summary; 2014 [cited 2019 May 16]. Available from: https:// www.mckinsey.com/industries/healthcare-systems-and-services/ our-insights/how-the-world-could-better-fight-obesity.

27. Mbochi RW, Kuria E, Kimiywe J, Ochola S, Steyn NP. Predictors 
of overweight and obesity in adult women in Nairobi Province, Kenya. BMC Public Health 2012;12:823.

28. French SA, Wall M, Mitchell NR. Household income difference in food sources and food items purchased. Int J Behav Nutr Phys Act 2010;7:77.

29. Rolls BJ. The relationship between dietary energy density and energy intake. Physiol Behav 2009;97:609-615.

30. El-Hazmi M, Warsy A. Relationship between age and the prevalence of obesity and overweight in Saudi population. Bahrain Med Bull 2002;24:1-7.

31. Dake FA, Tawiah EO, Badasu DM. Sociodemographic correlates of obesity among Ghanaian women. Public Health Nutr 2011;14:
1285-1291.

32. Duda RB, Darko R, Seffah J, Adanu RM, Anarfi JK, Hill AG. Prevalence of obesity in women of Accra, Ghana. Afr J Health Sci 2007; 14:154-159.

33. Yaya S, Ghosh S, Ghose B. Subjective happiness, health and quality of life and their sociocultural correlates among younger population in Malawi. Soc Sci 2019;8:55.

34. Ogana W. Food decisions and cultural perceptions of overweight and obesity: the case of Zulu women in Durban, South Africa; 2014 [cited 2019 Oct 14]. Available from: http://ukzn-dspace. ukzn.ac.za/bitstream/handle/10413/12719/Ogana_Winifred_2014. pdf ? sequence $=1$ \&isAllowed $=y$. 